\title{
Linking the rotation of a cluster to the spins of its stars: The kinematics of NGC 6791 and NGC 6819 in 3D
}

\author{
S. Kamann, ${ }^{1 \star}$ N. J. Bastian, ${ }^{1}$ M. Gieles,${ }^{2,3,4}$ E. Balbinot, ${ }^{2,5}$ V. Hénault-Brunet ${ }^{6}$ \\ ${ }^{1}$ Astrophysics Research Institute, Liverpool John Moores University, 146 Brownlow Hill, Liverpool L3 5RF, UK \\ ${ }^{2}$ Department of Physics, University of Surrey, Guildford, GU2 7XH, UK \\ ${ }^{3}$ Institut de Ciències del Cosmos (ICCUB), Universitat de Barcelona, Martí i Franquès 1, E08028 Barcelona, Spain \\ ${ }^{4}$ ICREA, Pg. Lluis Companys 23, 08010 Barcelona, Spain. \\ ${ }^{5}$ Kapteyn Astronomical Institute, University of Groningen, Landleven 12, 9747 AD Groningen, The Netherlands \\ ${ }^{6}$ National Research Council, Herzberg Astronomy 86 Astrophysics, 5071 West Saanich Road, Victoria, BC, V9E 2E7, Canada
}

Accepted XXX. Received YYY; in original form ZZZ

\begin{abstract}
The physics governing the formation of star clusters is still not entirely understood. One open question concerns the amount of angular momentum that newly formed clusters possess after emerging from their parent gas clouds. Recent results suggest an alignment of stellar spins and binary orbital spins in star clusters, which support a scenario in which clusters are born with net angular momentum cascading down to stellar scales. In this paper, we combine Gaia data and published line of sight velocities to explore if NGC 6791 and NGC 6819, two of the clusters for which an alignment of stellar spins has been reported, rotate in the same plane as their stars. We find evidence for rotation in NGC 6791 using both proper motions and line of sight velocities. Our estimate of the inclination angle is broadly consistent with the mean inclination that has been determined for its stars, but the uncertainties are still substantial. Our results identify NGC 6791 as a promising follow-up candidate to investigate the link between cluster and stellar rotation. We find no evidence for rotation in NGC 6819.
\end{abstract}

Key words: stars: kinematics and dynamics - open clusters and associations: individual: NG 6791, NGC 6819 - astrometry - techniques: radial velocities

\section{INTRODUCTION}

Recent results have suggested that the alignment of stellar rotation axes in clusters is not isotropically distributed, but rather has a preferential inclination angle (Corsaro et al. 2017). This result is based on asteroseismology analysis of Kepler data for stars in NGC 6791 and NGC 6819, two Galactic open clusters with ages of $\sim 8$ Gyr and $~ 2.4$ Gyr, respectively. While some caution needs to be taken in interpreting these results, due to potential systematic effects in inferring the stellar inclination angle from Kepler analyses (Kamiaka et al. 2018), the results potentially offer an unexpected and intriguing insight into the formation of stellar clusters. There has also been other studies that have found non-isotropic stellar orientations in clusters using photometric rotation periods and spectroscopic rotation velocities (e.g. Kovacs 2018).

In addition to individual stellar rotational orientations, alignment of binary orbital spins within clusters has been reported. Klagyivik et al. (2013) studied the young ( 3 Myr)

^ E-mail: skamann@ljmu.ac.uk
cluster/HiI region NGC 2264, and found that within the cluster the binary stars were preferentially seen edge-on (i.e. in eclipsing binaries) and that outside the cluster they were isotropically distributed.

The generally advocated explanation for the alignment of stellar rotation axes (and aligned binary orbital spins) in clusters is that the stars were born within a giant molecular cloud (GMC) that contained significant amounts of angular momentum, which was inherited by the stars as they formed (e.g. Corsaro et al. 2017; Rey-Raposo \& Read 2018). If the alignment of stars/binaries is strong at birth it persists within a cluster, as extremely close, i.e. rare, stellar dynamical interactions are required in order to change the orientation of the spin of a star. For tidal friction to be efficient, an encounter must be within a few stellar radii (e.g. Zahn 1977).

If either the stellar or the binary orientations are aligned, and if this is due to inheriting angular momentum from the progenitor GMCs, then we may expect that the host stellar cluster is itself rotating with the same orientation. Coherent cluster rotation has been found in clusters of all ages, from recently formed (e.g. Hénault-Brunet et al. 
2012) to intermediate age (e.g. Davies et al. 2011; Kamann et al. 2018b) and even in the ancient GCs (e.g. Bellazzini et al. 2012; Fabricius et al. 2014; Kamann et al. 2018a; Ferraro et al. 2018). Kamann et al. (2018a) further found a correlation between the angular momentum of the clusters and their relaxation times (see also Bianchini et al. 2018). As relaxation causes a cluster to lose angular momentum via transport of angular momentum and escaping stars (e.g. Einsel \& Spurzem 1999), this finding suggests that rotation is an intrinsic part of the cluster formation process. Such a scenario is also suggested by the detection of ordered motions in young H II regions (Dalgleish et al. 2018).

The feasibility of studying the rotation of star clusters with the Gaia DR2 catalogue (Gaia Collaboration et al. 2016, 2018b) has already been demonstrated by Bianchini et al. (2018) and Milone et al. (2018). Bianchini et al. (2018) further inferred inclination angles for several clusters by comparing to results obtained from line of sight (LOS) velocities. Here, we take advantage of the Gaia data and published LOS velocity measurements to look for evidence of cluster rotation in NGC 6791 and NGC 6819, two of the clusters with reported stellar rotation alignment. The goal is to see if the rotation axes of the clusters (if rotation is detected) align with the stellar rotational axes.

NGC 6791 ( $d=4.2 \mathrm{kpc}$, Basu et al. 2011) is a peculiar cluster given its old age $(\sim 8 \mathrm{Gyr}$, e.g., Grundahl et al. $2008)$ and high metallicity $([\mathrm{Fe} / \mathrm{H}]=+0.4$, e.g., Carraro et al. 2006). In light of these properties and its eccentric orbit, it has been suggested that it originated in the Galactic bulge (e.g. Bedin et al. 2006; Jílková et al. 2012). Dalessandro et al. (2015) observed tidal tails in NGC 6791 and concluded that the cluster must have initially been significantly more massive than its current mass of $5000 \mathrm{M}_{\odot}$.

NGC $6819(d=2.4 \mathrm{kpc}$, Basu et al. 2011) is considerably younger ( $2.4 \mathrm{Gyr}$, Rosvick \& Vandenberg 1998) than NGC 6791 and has about half of its mass $\left(2600 \mathrm{M}_{\odot}\right.$, Kalirai et al. 2001). Its metallicity is about solar (e.g. Bragaglia et al. 2001) and its orbit (Platais et al. 2013) consistent with an origin in the Galactic disk.

\section{DATA}

\subsection{Line of sight velocities}

Both clusters, NGC 6791 and NGC 6819, have been targeted by the WIYN open cluster study (WOCS, Mathieu 2000). We made use of the published catalogues which include the average LOS velocities of the target stars, as well as their uncertainties and variability flags.

Tofflemire et al. (2014) provide data for 280 stars towards NGC 6791. While no uncertainties are provided for the velocities of the individual stars, they can be recovered from the average uncertainty of $0.38 \mathrm{~km} \mathrm{~s}^{-1}$ and the ratios between the standard deviation of each star and the overall precision $(e / i$, cf. Table 1 in Tofflemire et al. 2014). After excluding stars flagged as non-members, possible members, and binary candidates, we were left with a sample of 101 stars.

For NGC 6819, we combined two WOCS studies, namely Hole et al. (2009) and Lee-Brown et al. (2015), providing accurate velocities for 1207 and 333 stars, respec- tively. For 304 stars that we found to be present in both studies (based on a spatial offset $<10^{-3}$ arcmin) we obtained combined LOS velocities using weighted averages of the individual measurements. The standard deviation between the catalogues (after correcting for a global offset of $0.23 \mathrm{~km} \mathrm{~s}^{-1}$ ) was $1.06 \mathrm{~km} \mathrm{~s}^{-1}$, in line with the mean uncertainty of $0.79 \mathrm{~km} \mathrm{~s}^{-1}$ that we obtained for the combined velocities. Exclusion of non-members, possible members, and binary stars resulted in a final sample of 397 LOS velocities for NGC 6819.

\subsection{Gaia photometry and astrometry}

We selected all stars available in Gaia DR2 that lie within a radius of $20^{\prime}$ from any of the two cluster centres. The size of the selection radius was motivated by the spatial extents of the clusters on the sky. For NGC 6791, Platais et al. (2011) find a tidal radius of $23.1^{\prime}$ using King profile fits and for NGC 6819, a Roche radius of $23^{\prime}$ has been determined by Kalirai et al. (2001).

To select cluster members, each star was assigned a membership probability based on its parallax and proper motion measurements. In brief, for each of the three quantities $\left(x \in\left\{\varpi, \mu_{\alpha}^{*}, \mu_{\delta}\right\}\right)$, we defined a Gaussian probability density,

$$
p(x)=\frac{1}{\sqrt{2 \pi\left(\epsilon_{x}^{2}+\sigma_{x}^{2}\right)}} \exp \left\{-\frac{(x-\bar{x})^{2}}{2\left(\epsilon_{x}^{2}+\sigma_{x}^{2}\right)^{2}}\right\},
$$

where $\epsilon_{x}$ indicates the measurement uncertainty from Gaia, $\bar{x}$ is the mean value of the cluster, and $\sigma_{x}$ the intrinsic dispersion of the cluster. Cluster parallaxes were obtained by inverting the (uncorrected) distance moduli from Choi et al. $(2018),(m-M)=13.29($ NGC 6791$)$ and $12.22\left(\right.$ NGC 6819) ${ }^{1}$ We neglected the (small) intrinsic dispersions caused by the finite cluster widths, i.e. set $\sigma_{\varpi}=0$. Further, we determined initial guesses for the systemic motions of the clusters visually by searching for overdensities of the full Gaia samples in proper motion space. The initial guesses were iteratively refined after a preliminary member selection had been performed and the final values are provided in Table 1. They are in good agreement with previous estimates of the systemic proper motions of the clusters (e.g. Bedin et al. 2006; Platais et al. 2013). As intrinsic dispersion, we assumed $1 \mathrm{~km} \mathrm{~s}^{-1}$, comparable to the virial estimates of both clusters.

Each star with a combined membership probability of $p(\varpi) \times p\left(\mu_{\alpha}^{*}\right) \times p\left(\mu_{\delta}\right)>0.01$ was considered a cluster member. In addition, we only kept stars with precisely measured proper motions, corresponding to uncertainties $<4 \mathrm{~km} \mathrm{~s}^{-1}$ and $<2 \mathrm{~km} \mathrm{~s}^{-1}$ at the distances of NGC 6791 and NGC 6819, respectively. These error cuts were chosen as a compromise between obtaining sufficiently large samples of stars while avoiding contamination of our samples with stars having poorly determined kinematics. When using less stringent error cuts, we started to observe contamination by field stars in the colour-magnitude diagrams (cf. Figs 1 and 2). Note that the chosen error cuts correspond to approximately the same accuracy in proper motion space because the distance

1 Note that the distance moduli were determined by Choi et al. (2018) from the Gaia parallaxes of their cluster members, hence were not corrected for the systematic offset of $-0.029 \mathrm{mas} / \mathrm{yr}$ in DR2 (Lindegren et al. 2018). 


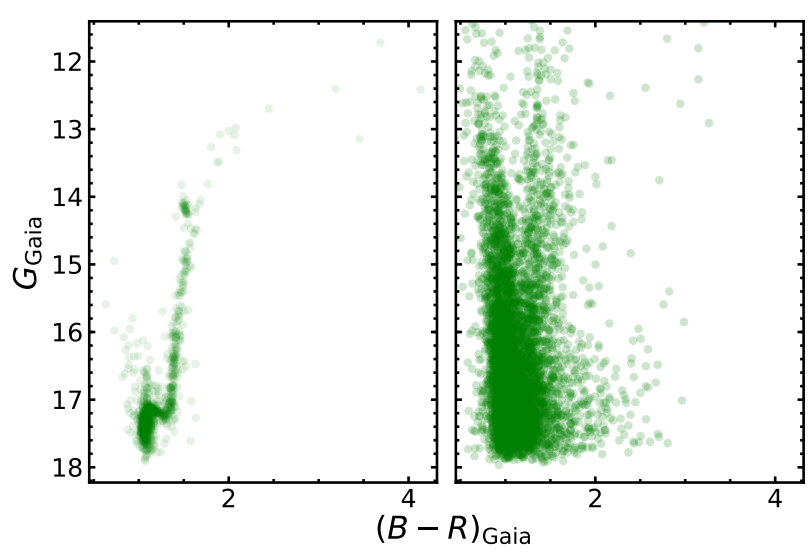

Figure 1. Gaia colour-magnitude diagrams for stars in direction of NGC 6791 (within a distance of 20' of the assumed cluster centre), separated into cluster members (left) and field stars (right).

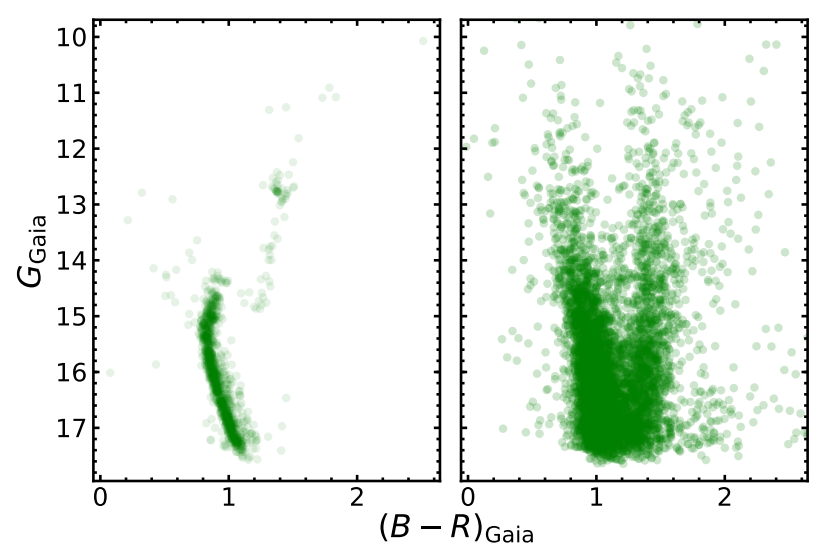

Figure 2. Same as Fig. 1 for NGC 6819.

of NGC 6791 is about a factor of 2 larger than that of NGC 6819 .

Our member selection resulted in samples of 964 and 1244 stars for NGC 6791 and NGC 6819, respectively. In order to illustrate the success of our membership selection, we show colour-magnitude diagrams of both clusters, split up into members and foreground stars, in Figs 1 and 2.

Finally, we note that DR2 contains only few LOS velocities for cluster members (6 in NGC 6791 and 35 in NGC 6819). Therefore, we did not include Gaia LOS velocities in our analysis.

\section{CLUSTER MORPHOLOGIES}

The catalogs of cluster members created from the Gaia data allow us to determine the exact locations of the cluster centres and to study the two-dimensional density structures of the clusters. The latter is important because dynamical models usually require some assumptions on the morphology of the system (such as an axisymmetric cluster, see van de Ven et al. 2006).
Table 1. Cluster properties derived in this work.

\begin{tabular}{ccc}
\hline & NGC 6791 & NGC 6819 \\
\hline$\alpha(\mathrm{J} 2000)$ & $19: 20: 51.3$ & $19: 41: 17.2$ \\
$\delta(\mathrm{J} 2000)$ & $+37: 46: 26$ & $40: 11: 18$ \\
$\mu_{\alpha}^{*}[\mathrm{mas} / \mathrm{yr}]$ & -0.42 & -2.91 \\
$\mu_{\delta}[\mathrm{mas} / \mathrm{yr}]$ & -2.27 & -3.86 \\
$r_{\mathrm{hl}}[\mathrm{arcmin}]$ & $4.10 \pm 0.16$ & $5.12 \pm 0.28$ \\
\hline
\end{tabular}

\subsection{Cluster centres}

In order to determine the locations of the cluster centres, we proceeded as follows. For each cluster, we defined a grid of $50 \times 50$ points of trial centres, with a total length of 2 arcmin on each side, around the centre positions available in SIMBAD. At each grid point, we selected the stars from our Gaia samples within a distance of 8 arcmin and split them up into eight equally sized pie slices, each covering an angle of $\pi / 4$. Then we compared the number of stars in the pie slices opposite to each other. Under the hypothesis that a grid point represents the true centre of a cluster, each star has equal probability of falling into either of the two slices. Hence, the number ratio of stars between the two slices would follow a binomial distribution and we can assign each grid point a probability of representing the cluster centre. The actual centre of each cluster was then obtained by fitting a twodimensional Gaussian to the distribution of probabilities. The central coordinates we found this way are provided in Table 1.

\subsection{Ellipticities and position angles}

With the centres of the clusters in place, we proceeded by constraining their ellipticities $e$ and position angles $\theta$. The latter provide the orientations of the semi-major axes and are measured from north through east, i.e. in counterclockwise direction. We followed Fabricius et al. (2014) and first determined the eigenvalues $\lambda_{1,2}$ and eigenvectors $\vec{v}_{1,2}$ of the two-dimensional distributions of Gaia members of each cluster. The eigenvalues are related to the lengths of the semi-major and semi-minor axes via $\lambda_{1}=1 / a^{2}$ and $\lambda_{2}=1 / b^{2}$. Hence, the ellipticity $e$ of each cluster is given as $e=1-\sqrt{\lambda_{1} / \lambda_{2}}$. The eigenvector $\vec{v}_{2}$ points in the direction of the semi-major axis.

As the impact of external effects such as tidal forces will vary with distance to the cluster centres, we calculated the morphologies of NGC 6791 and NGC 6819 in different radial bins. The results of this calculation are shown in Fig. 3. The uncertainties included in Fig. 3 were estimated by considering two effects that could potentially impact our measurements, namely uncertainties in our determination of the cluster centres and the finite number of stars per radial bin. We checked the influence of the former by varying the cluster centres within a radius of 5 arcsec (the approximate uncertainty of our centre determination) and recalculating the eigenvalues and eigenvectors. We found that our measurements were hardly affected by the exact locations of the cluster centres.

On the other hand, the finite number of stars seems to be the dominant source of uncertainty. To measure its influence, we created radial star count profiles and fitted them 

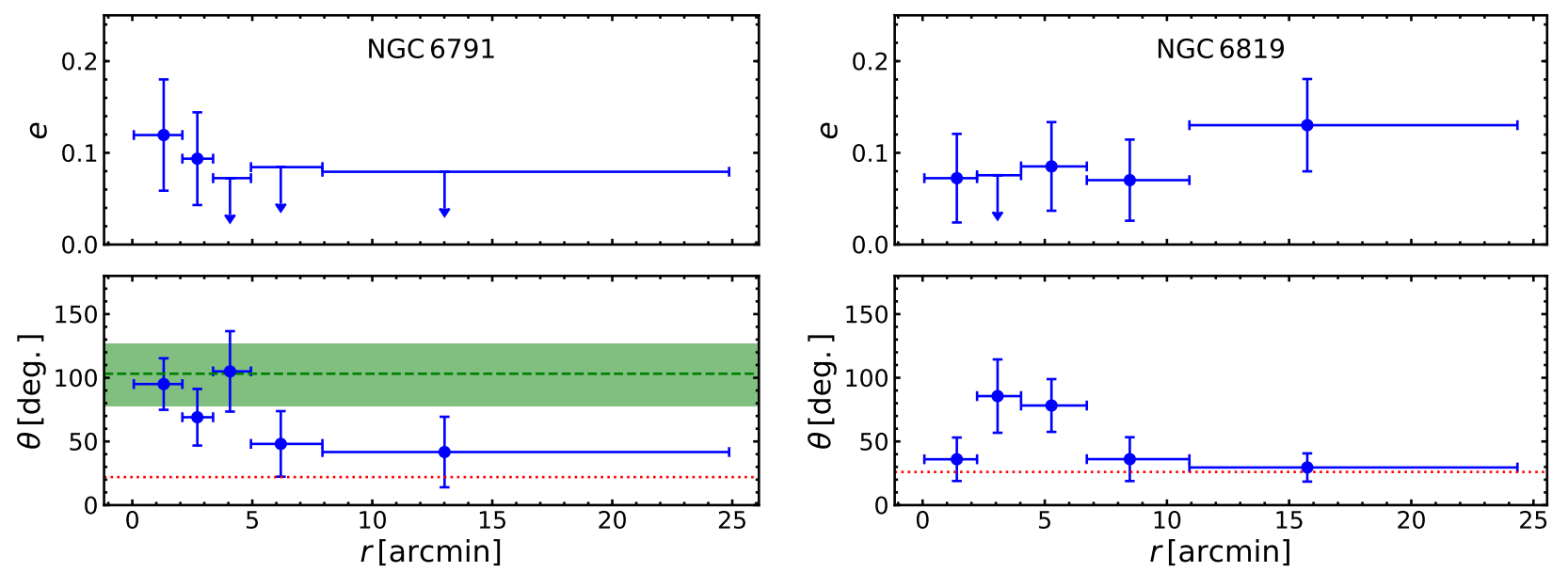

Figure 3. Ellipticity (top) and position angle of the semi-major axis (bottom, measured from north through east) of NGC 6791 (left) and NGC 6819 (right) as a function of distance to the cluster centre. The dotted red lines in the bottom panels indicate the directions towards the Galactic Centre. For NGC 6791, we also show the position angle expected from the analysis of the LOS velocities (green dashed line, cf. Sect. 4.1). Horizontal error bars show the limits of the radial bins.

with multiple Gaussians (using the code of Cappellari 2002). Then we created mock Gaia samples by randomly drawing stars from the multi-Gaussian model and assigning each star a random position angle from the interval $[0,2 \pi)$. For each of the mock samples, we determined the ellipticity and position angle. The standard deviations of the two distributions thus obtained served as estimates of the parameter uncertainties due to the finite number of stars. From the radial profiles, we further estimated half-light radii of the two clusters. They are included in Table 1.

We include in the lower panels of Fig. 3 the directions towards the Galactic Centre, $\theta_{\mathrm{GC}}$, as dashed red lines. ${ }^{2}$ In the bottom right panel of Fig. 3, it can be seen that for NGC 6819 there is a general agreement between $\theta_{\mathrm{GC}}$ and the position angle of the semi-major axis, indicating that the cluster is stretched along a line passing through the cluster and the Galactic Centre. The elongation further seems to increase with distance to the cluster centre, with the outermost bin having an ellipticity of $e \sim 0.15$, whereas $e<0.1$ in the inner four bins, although the significance of this trend is low. Both of these effects, the stretch towards the Galactic Centre and its increase in the cluster outskirts, are expected for star clusters evolving in the Galactic tidal field (Terlevich 1987; Portegies Zwart et al. 2001; Chen et al. 2004). The strengths of these effects is expected to increase with cluster age. However, NGC 6791, which is significantly older than NGC 6819, does show a different behaviour. Its position angle is significantly offset from the direction towards the Galactic Centre and its ellipticity seems to decrease with radius. This suggests that other effects, such as internal rotation or pressure anisotropies, impact the shape of NGC 6791 (e.g. van den Bergh 2008).

At first sight, our results for NGC 6791 seem in tension with the findings of Dalessandro et al. (2015), who observed

2 Note that for both clusters, the Galactic Centre is actually located at $\theta_{\mathrm{GC}}-\pi$. However, our method to determine $e$ and $\theta$ is invariant to angular offsets of $\pi$. a clear elongation of NGC 6791 towards the Galactic Centre using ground based data. However, their Fig. 5 shows that this elongation is restricted to distances $>500^{\prime \prime}$ from the cluster centre, a radial regime in which our sample is lacking sufficient stars for a detailed analysis. At distances $<500^{\prime \prime}$, Dalessandro et al. (2015) observe a shift of the position angle by $\sim 90$ degrees, in good agreement with the behaviour visible in Fig. 3. We will revisit the peculiar elongation pattern of NGC 6791 in the context of cluster rotation below.

\section{CLUSTER DYNAMICS}

Our analysis of the cluster dynamics is carried out in several steps. First, we analyse the LOS velocities and the proper motions of each cluster separately. If evidence for rotation is found, we then combine the results obtained from the two analyses to infer an inclination angle. In order to verify our approach, we perform the same analysis on a mock data set, extracted from an $\mathrm{N}$-body model of a rotating cluster. The latter is described in detail in Appendix A. Briefly, the mock data is based on the final snapshot of an $N$-body model of a rotating cluster evolved to an age of 10.75 Gyr, for which the initial dimensionless spin parameter $\lambda$ (Peebles 1969) was set to $\lambda=0.091$ (model MGEN1 in Table 1 of HénaultBrunet et al. 2015), corresponding to about $10 \%$ of the total kinetic energy of the cluster in rotation at the start of the simulation.

\subsection{Line of sight velocities}

We followed the approach outlined in Kamann et al. (2018a) to obtain maximum-likelihood estimates of the systemic velocity $v_{0}$, the velocity dispersion $\sigma_{\mathrm{LOS}}$, the rotation velocity $v_{\text {rot }}$, and the position angle $\theta_{0}$ of the rotation axis for each cluster at the same time. In brief, it works by maximizing 

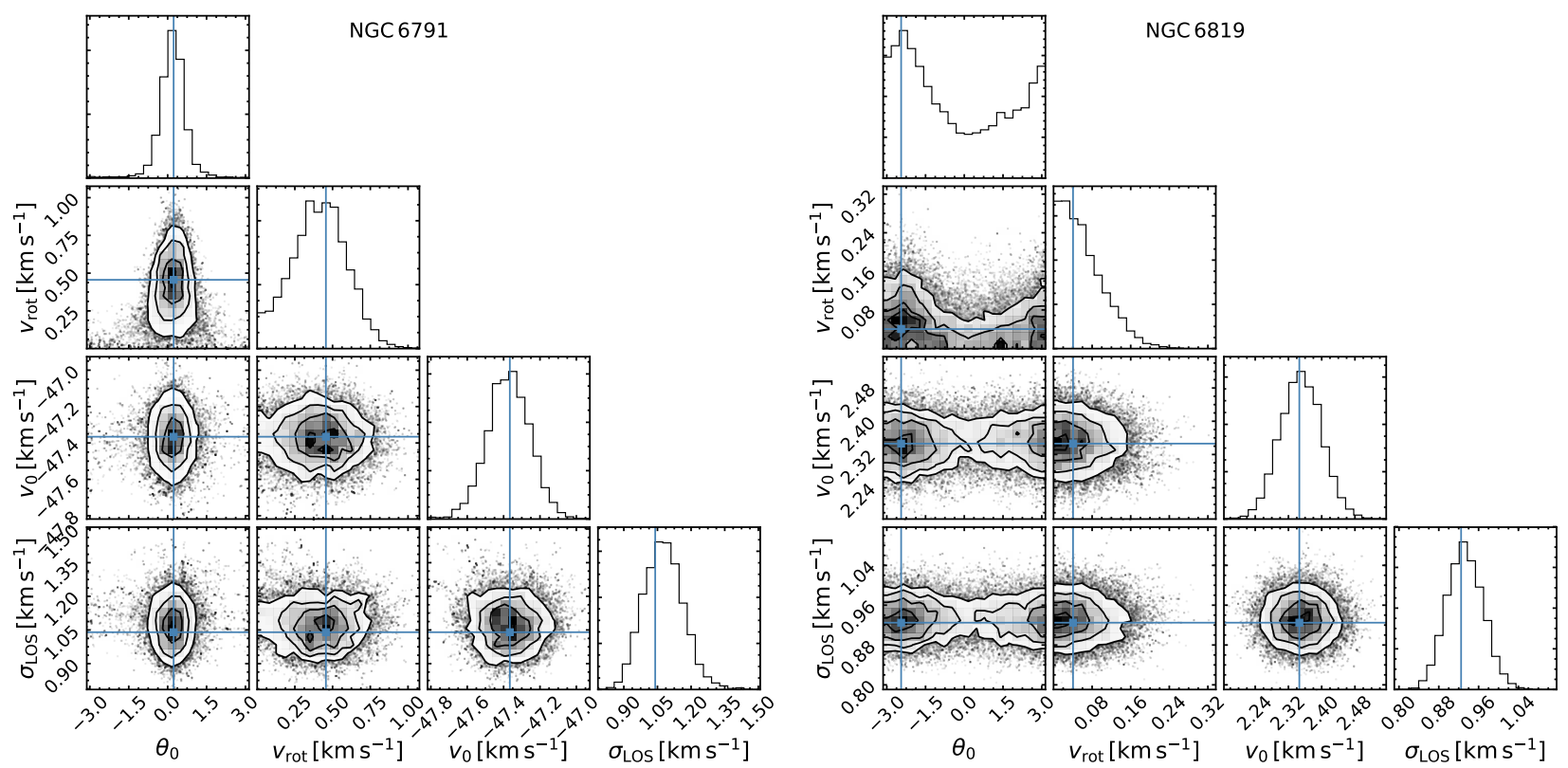

Figure 4. Kinematic cluster parameters of NGC 6791 (left) and NGC 6819 (right) obtained from an MCMC analysis of the LOS velocities as described in the text. The top panel of each column shows the 1D histogram of parameter values sampled by the MCMC walkers, whereas subjacent panels show 2D distributions of parameter pairs. The median value of each parameter is indicated by a solid blue line.

the likelihood $\mathcal{L}$, calculated according to,

$\ln \mathcal{L}=-\sum_{i=1}^{n} \ln \left(2 \pi \sqrt{\sigma_{\mathrm{LOS}}^{2}+\epsilon_{i}^{2}}\right)-\frac{\left(v_{i}-v_{\mathrm{c}}\right)^{2}}{2\left(\sigma_{\mathrm{LOS}}^{2}+\epsilon_{i}^{2}\right)}$,

where $v_{\mathrm{c}}=v_{0}+v_{\text {rot }} \sin \left(\theta_{i}-\theta_{0}\right)$ is the systemic cluster velocity corrected for the effect of rotation. For each of the $n$ sources entering the above equation, the position angle $\theta_{i}$, the LOS velocity $v_{i}$, and its uncertainty $\epsilon_{i}$ must be known. Note that equation (1) assumes constant dispersion and rotation curves. However, the generalization to spatially varying curves can be easily accomplished using analytical functions that evaluate $\sigma_{\mathrm{LOS}}\left(R_{i}, \theta_{i}\right)$ and $v_{\text {rot }}\left(R_{i}, \theta_{i}\right)$ for each stellar position, where $\left(R_{i}, \theta_{i}\right)$ are the positions of the stars on the plane of the sky. Yet in view of the relatively small sample sizes, especially for NGC 6791, we did not try to obtain radial profiles for the various parameters but rather concentrated on obtaining global values. A caveat with this approach is that the global values could be biased towards the dispersion and rotational amplitude in the region of the cluster best sampled by the data. However, the half-number radii of our LOS velocity samples are 4.1' (NGC 6791) and 4.8' (NGC 6819), respectively, very similar to the half-light radii of the clusters (cf. Table 1 ). Therefore, we do not expect that assuming a constant rotation curve is dragging our results in a certain direction because of preferential coverage.

Prior to the analysis, we corrected the LOS velocities for the effect of perspective rotation, using the systemic proper motions of the clusters listed in Table 1 and the method outlined in van de Ven et al. (2006). The corrections we applied to the individual velocities reached from $-0.15 \mathrm{~km} \mathrm{~s}^{-1}$ to $0.27 \mathrm{~km} \mathrm{~s}^{-1}$ for NGC 6791 and from $-0.20 \mathrm{~km} \mathrm{~s}^{-1}$ to $0.19 \mathrm{~km} \mathrm{~s}^{-1}$ for NGC 6819 .

In order to assign uncertainties to each parameter, we used the code EMCEE of Foreman-Mackey et al. (2013) and obtained the 16th and 84th percentiles of the parameter posterior probability distributions resulting from $100 \mathrm{MCMC}$ walkers with 500 steps each. For both clusters, best-fit values (defined as the median values of the posterior distributions) and the uncertainties of the four model parameters are provided in Table 2. Further, Fig. 4 shows corner plots of the parameter distributions returned by the MCMC sampler. The plots were created using the code of Foreman-Mackey (2016).

In NGC 6791, we detect rotation at the $2.2 \sigma$ level, around an axis offset by 14.2 degrees from north in counterclockwise direction. The rotation velocity of $0.40 \mathrm{~km} \mathrm{~s}^{-1}$ corresponds to about 37 per cent of the measured velocity dispersion. This $v_{\text {rot }} / \sigma_{\text {LOS }}$ ratio is similar to the one we find in the analysis of the mock data used to validate our fitting method (cf. Appendix A, see Fig. A1).

To investigate if the rotation of NGC 6791 leaves an imprint in the morphology of the cluster, we overplot in the lower left panel of Fig. 3 the position of the semi-major axis one would expect if the elongation of the cluster was caused by rotation ${ }^{3}$. The elongation of the inner three bins agrees very well with this expectation whereas the orientation of the outer bins is intermediate between those expected from rotation and tidal forces. So the inner parts of NGC 6791 appear to behave as an oblate rotator while tides may also influence the shape of the cluster beyond $5^{\prime}$.

On the other hand, we do not find any signs of rotation along the line of sight in NGC 6819, despite the fact that our sample of stars is almost 4 times as large compared to

3 Note that for an oblate rotator the semi-major axis is perpendicular to the rotation axis. This seems to be the case for most globular clusters (Fabricius et al. 2014; Kamann et al. 2018a). 
Table 2. Dynamical cluster parameters derived from the line of sight velocities.

\begin{tabular}{ccc}
\hline & NGC 6791 & NGC 6819 \\
\hline$v_{0}\left[\mathrm{~km} \mathrm{~s}^{-1}\right]$ & $-47.37_{-0.12}^{+0.11}$ & $2.35_{0.05}^{0.05}$ \\
$\sigma_{\text {LOS }}\left[\mathrm{km} \mathrm{s}^{-1}\right]$ & $1.07_{-0.08}^{+0.10}$ & $0.93_{0.04}^{0.04}$ \\
$v_{\text {rot }}\left[\mathrm{km} \mathrm{s}^{-1}\right]$ & $0.40_{-0.18}^{+0.17}$ & $0.05_{0.04}^{0.06}$ \\
$\theta_{0}[\mathrm{deg}]$. & $14_{-26}^{+24}$ & $-153_{-98}^{+919}$ \\
\hline
\end{tabular}

NGC 6791. Instead, we obtain a very stringent upper limit of $0.11 \mathrm{~km} \mathrm{~s}^{-1}$ for the rotation amplitude, cf. Table 2. Given the similarity in $\sigma_{\text {LOS }}$ for the two clusters, this implies that either NGC 6819 has a lower intrinsic rotational velocity, or its rotation is more in the plane of the sky. We will address this in the next section.

\subsection{Gaia proper motions}

To study the cluster dynamics using the Gaia data, we defined a Cartesian coordinate system for each cluster, with its origin on the cluster centre, $x$ increasing westwards, and $y$ increasing northwards. The world coordinates and proper motions from the Gaia catalog were transformed into the new coordinate system using standard formulas (see, e.g., Gaia Collaboration et al. 2018a). Afterwards, we converted the relative proper motions to polar coordinates. The formulas for this conversion can be found in, e.g., van Leeuwen et al. (2000). The uncertainties of $\mu_{\alpha}^{*}$ and $\mu_{\delta}$ were propagated during this process while possible covariances between the five astrometric parameters were neglected.

Polar coordinates offer a convenient way to study rotation using proper motions because the intrinsic rotation is mainly contained in the tangential component while the perspective expansion or contraction effect caused by the LOS velocity of the system only affects the radial component. Hence, in order to obtain the cluster kinematics, we applied a similar maximum-likelihood approach as for the LOS velocities (cf. Sect. 4.1) independently to the tangential and the radial proper motion component. However, this time we only solved for the mean value $\langle\mu\rangle$ and the dispersion $\sigma_{\mu}$ of each component, i.e. set $v_{\mathrm{c}}=v_{0}$ in equation (1), as even for a rotating cluster no sinusoidal velocity variation with position angle is expected. Instead, the mean value of the tangential component corresponds to the rotation velocity in the plane of the sky. Thanks to the samples of $\sim 1000$ stars with reliable proper motion measurements available in Gaia DR2, we were able to create radial profiles of the proper motion kinematics. They are displayed in Fig. 5.

In the upper panels of Fig. 5, dashed green lines indicate the expected expansion or contraction of each cluster given its systemic velocity. The predictions were obtained by expressing the first two lines of equation 6 in van de Ven et al. (2006) in polar coordinates. The mean values of the radial component in NGC 6791 are in reasonable agreement with the prediction. A mild expansion is observed, as expected given its approaching LOS velocity of $-47.4 \mathrm{~km} \mathrm{~s}^{-1}$. For NGC 6819, a very small contraction is expected from its receding LOS velocity of $2.4 \mathrm{~km} \mathrm{~s}^{-1}$. However, the contraction that we observe is stronger. An almost constant offset of $\sim 0.01 \pm 0.01 \mathrm{mas} \mathrm{yr}^{-1}\left(\sim 0.1 \pm 0.1 \mathrm{~km} \mathrm{~s}^{-1}\right)$ is visible in the upper right panel of Fig. 5. The reason for this behaviour is currently unknown, but it seems unlikely that the cluster is actually contracting. More likely, we are looking at systematic effects in the Gaia data. We will revisit this issue in Sect. 4.4 below.

The radial profiles of the mean tangential proper motions depicted in the upper panels of Fig. 5 confirm the results found using the LOS velocities. While no significant deviations from zero are observed for NGC 6819, the mean values in NGC 6791 show a trend towards negative values at distances $>4^{\prime}$ to the cluster centre. This trend corresponds to clockwise rotation.

The mean tangential proper motion outside of $4^{\prime}$ is

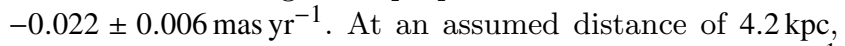
this corresponds to a rotation velocity of $0.42 \pm 0.12 \mathrm{~km} \mathrm{~s}^{-1}$ on the sky, i.e. comparable to the rotational amplitude seen in the line-of-sight velocities. However, this comparison neglects that we assumed a constant rotation velocity when analysing the LOS velocities. If we do the same for the proper motions, we get a mean tangential velocity of $-0.007 \pm 0.004 \mathrm{mas} \mathrm{yr}^{-1}$, corresponding to $0.14 \pm 0.08 \mathrm{~km} \mathrm{~s}^{-1}$.

Interestingly, the radial trend observed for the proper motions seems to be absent in the LOS velocities. If we split the latter sample in two subsamples according to the distance to the cluster centre (lower and higher than 4 arcmin), both subsamples yield a rotation velocity of around $0.4 \mathrm{~km} \mathrm{~s}^{-1}$. However, the sample sizes are small, so that a potential radial trend may also be hidden in the noise. Finally, we note that no rotation signal is detected in the proper motions of our LOS velocity stars (83/101 stars are in the Gaia sample). The $1 \sigma$ upper limit of $0.008{\mathrm{mas} \mathrm{yr}^{-1}}^{-1}$ that we find corresponds to $0.16 \mathrm{~km} \mathrm{~s}^{-1}$.

We show the velocity dispersion profiles in the lower panels of Fig. 5. Also shown in those panels are the velocity dispersions obtained during the analysis of the LOS velocity samples (cf. Sec. 4.1) and predicted using simple virial estimates (eq. 4.249b in Binney \& Tremaine 2008),

$\left\langle v^{2}\right\rangle=\frac{0.45 G M_{\mathrm{c}}}{r_{\mathrm{hl}}}$,

with $\left\langle v^{2}\right\rangle=3 \sigma^{2}$. We assumed cluster masses of $M_{\mathrm{c}}=$ $5000 \mathrm{M}_{\odot}\left(\mathrm{NGC} 6791\right.$, Dalessandro et al. 2015) and $2600 \mathrm{M}_{\odot}$ (NGC 6819, Kalirai et al. 2001). The half-light radii $r_{\mathrm{hl}}$, listed in Table 1, were derived from the star count profiles discussed in Sect. 3.2. The virial estimate agrees reasonably well with the measured dispersion in NGC 6791 and underestimates our measurements in NGC 6819. Such an offset could be caused by underestimated velocity uncertainties. The median uncertainties of our proper motion samples correspond to $2.4 \mathrm{~km} \mathrm{~s}^{-1}$ (NGC 6791) and $0.8 \mathrm{~km} \mathrm{~s}^{-1}$ (NGC 6819) and are comparable to the intrinsic dispersions, so they can potentially impact our dispersion measurements. However, for both clusters we find good agreement with the dispersion values determined from the LOS velocity data, indicating that the uncertainties for the Gaia proper motions are fairly accurate.

Nevertheless, deviations due to inaccurate uncertainties are still likely. An indication in this regard may be the lack of a decrease of the dispersion measurements with distance to the cluster centres, as one would expect for a relaxed cluster. Alternatively, a significant contribution from energetically unbound stars (so-called potential escapers, Küpper et al. 

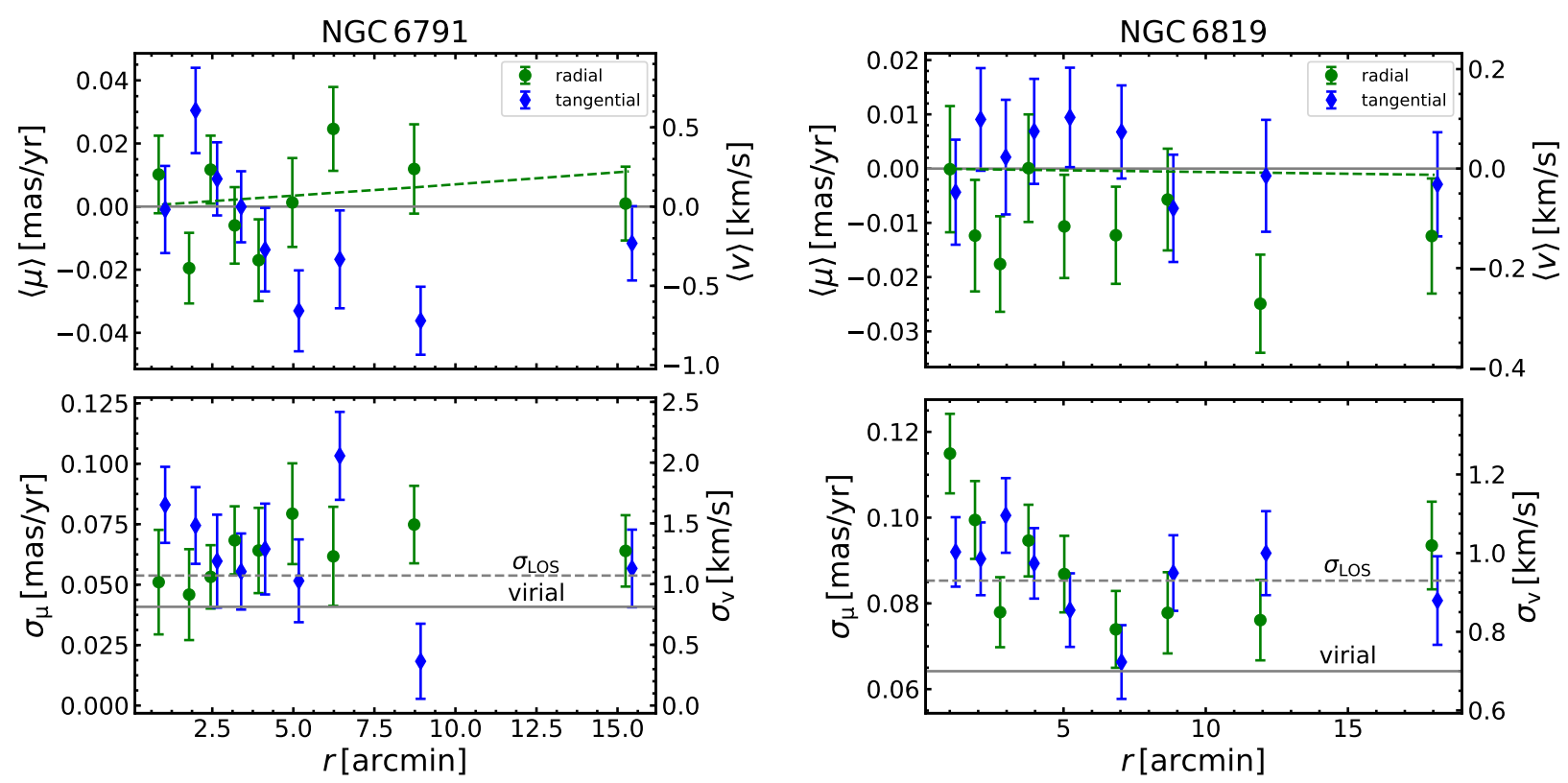

Figure 5. Proper motion kinematics of NGC 6791 (left) and NGC 6819 (right). The radial profiles of the mean velocities are shown in the top panels while the bottom panels show the velocity dispersion profiles as a function of distance to the cluster centres. Blue diamonds show the results obtained for the tangential components while green circles are used for the results obtained for the radial components. In the upper panels, a dashed green line indicates the expected behaviour of the radial component according to the LOS velocity of the cluster (perspective contraction or expansion). The grey solid line in the lower panels indicates the virial estimate of the velocity dispersion of each cluster while the grey dashed line indicates the dispersion value obtained from the LOS velocities. The distance moduli of NGC 6791 and NGC 6819 provided by Basu et al. (2011) have been used to convert the measured proper motions from mas yr ${ }^{-1}$ to $\mathrm{km} \mathrm{s}^{-1}$ as indicated on the right $\mathrm{y}$-axis of each panel.

2010; Claydon et al. 2017; Daniel et al. 2017) could explain this observation. In fact, the $N$-body models of NGC 6791 by Martinez-Medina et al. (2018) show that the velocity dispersion profile in projection decreases only by about 30 per cent in the central $\sim 7^{\prime}$ and stays almost constant beyond this radius. Note that we observe a similar behaviour in our analysis of the mock data presented in Appendix A, (see Fig. A2).

Given the small internal velocities and potential residual errors in the Gaia data, it is not possible to use the ratio between the tangential and radial dispersion profiles to make robust statements about possible anisotropies in the clusters. We only note that the ratios inferred from Fig. 5 are essentially all consistent with one, suggesting isotropy, as is expected for a cluster in the late stages of its evolution (e.g. Zocchi et al. 2016).

\subsection{Inclination angles}

Given the absence of any significant rotation signal in NGC 6819, we focus on NGC 6791 in our efforts to constrain the inclination angle of the rotation field. As a first estimate, we simply determined the arcus tangent of the ratio of the rotation velocities along the line of sight $\left(0.40 \pm 0.18 \mathrm{~km} \mathrm{~s}^{-1}\right.$, cf. Table 2) and in the plane of the sky $\left(-0.14 \pm 0.08 \mathrm{~km} \mathrm{~s}^{-1}\right.$, cf. Sec. 4.2). In doing so, we essentially treat the cluster as a rotating disk, seen at an inclination angle $i$. The comparison yields an inclination angle of $i=73_{-28}^{+11} \mathrm{deg}$ and hence suggests a strongly inclined rotation field. ${ }^{4}$ To obtain a conservative lower limit for the inclination, we recalculate it using the mean tangential proper motion measured at distances $>4^{\prime}$ to the cluster centre, $0.42 \pm 0.12 \mathrm{~km} \mathrm{~s}^{-1}$. This yields $i=43 \pm 15$ deg.. Finally, we also checked what happens if we restrict our proper motion sample to those stars that also have LOS velocity measurements. As already mentioned in Sec. 4.2, no rotation is obvious in their proper motion distribution. Accordingly, we obtain a lower limit on the inclination angle of $i>68$ degrees.

A more sophisticated way to determine the inclination of the rotation field of a cluster was used by van de Ven et al. (2006). They used a relation originally found by Evans \& de Zeeuw (1994) between local means of the proper motion along the semi-minor axis $\left\langle\mu_{\mathrm{v}}\right\rangle$ and the LOS velocity $\left\langle v_{\mathrm{r}}\right\rangle$ of an axisymmetric system,

$\left\langle v_{\mathrm{r}}\right\rangle=4.74 d \tan i\left\langle\mu_{\mathrm{v}}\right\rangle$,

where $d$ is the distance in kpc. In Sections 3 and 4.1, we found that the observed position angle of the photometric semi-major axis of NGC 6791 agrees reasonably well with the expectation for an oblate rotator. Hence, we assume in the following that the semi-major axis of NGC 6791 is perpendicular to the rotation axis, for which the position angle

\footnotetext{
${ }^{4}$ Note that $i$ can be positive or negative, corresponding to counterclockwise or clockwise rotation in the plane of the sky. However, in the work by Corsaro et al. (2017) only positive spin inclinations are considered, hence we also restrict ourselves to $i>0$.
} 
is provided in Table 2. Under this assumption, we can rotate our Cartesian coordinate system into a new system $(u$, $v$ ) such that $u$ is aligned with the semi-major axis of the cluster. Following van de Ven et al. (2006), we then defined a polar grid in the new coordinate system and binned the data according to their coordinates in the grid. Before binning the data, we exploited the symmetries of the system to transform all velocity measurements to the first quadrant of the $(u, v)$ coordinate system. ${ }^{5}$ Given the relatively small number of LOS velocity measurements in NGC 6791, we selected the bin sizes such that each bin contained on average 10 such measurements.

Figure 6 shows the relation between the mean minoraxis proper motion and the mean LOS velocity for each bin in the polar grid. Based on our previous analyses, the mean LOS velocities are expected to be $>0$, while the mean minoraxis proper motions are expected to be $<0$ (corresponding to clockwise rotation). While the former can be verified from Fig. 6, we only see mild evidence for the latter. However, a more obvious trend might be hidden under the noise given that the means in all bins are consistent with negative values within their uncertainties.

We used an orthogonal distance regression method to fit the relation defined in eq. 3 to the data shown in Fig. 6 . The method takes into account the uncertainties in both coordinates. We forced the fit to go through the centre of the coordinate system. It yielded an inclination angle of $45.4_{-18.0}^{+11.1}$ degrees.

For the stellar spins in NGC 6791, Corsaro et al. (2017) found a mean inclination angle of $30 \mathrm{deg}$., with the most inclined stars having values of $\sim 45 \mathrm{deg}$.. Thus all of our estimates suggest that the rotation of the cluster is more strongly inclined than the mean stellar spin. However, some of our estimates are still consistent with $i=30 \mathrm{deg}$. within their uncertainties. In particular, this is the case for the value of $i$ we obtained using eq. 3 and our analysis of the mock data (cf. Appendix A) suggests that this value is the most robust. Therefore we cannot exclude the possibility that the cluster does rotate in the same plane that has been reported for the stars by Corsaro et al. (2017).

The uncertainties in our inclination estimates are still substantial. One reason for this is the relatively low number of LOS velocities, so that each bin in Fig. 6 contains only a few stars with such measurements. Accordingly, the uncertainties of the mean values are still comparable to the expected rotation signal. While the mean proper motions suffer much less from low number statistics, the uncertainties of the mean values derived from them are only a factor $\lesssim 2$ lower. The reason is that the uncertainties of each measurement are larger than for the LOS velocities. This will likely improve with future Gaia data releases, as the end-ofmission uncertainties for Gaia proper motions are expected to be lower by a factor of $\geq 5$ compared to DR2.

\footnotetext{
5 In an axisymmetric system, LOS velocities and minor-axis proper motions are symmetric to reflections about the semi-major axis and antisymmetric to reflections about the semi-minor axis. Major-axis proper motions are antisymmetric to reflections about the semi-major axis and symmetric to reflections about the semiminor axis.
}

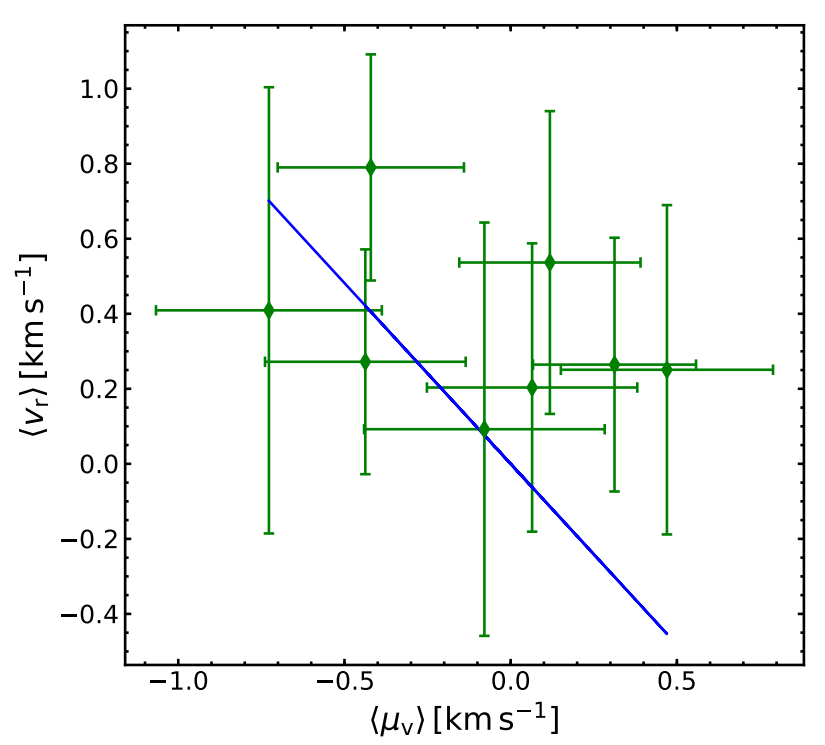

Figure 6. Relation between the mean proper motion along the assumed semi-minor axis and the mean LOS velocity for NGC 6791. The mean values have been obtained by binning the data in on a polar grid. The blue line shows a linear fit to the data, constrained to pass through the origin.

\subsection{Systematic effects}

The Gaia astrometric data suffers from well documented systematics (e.g. Lindegren et al. 2018; Gaia Collaboration et al. 2018a; Bianchini et al. 2018). In order to verify if they have the potential to impact our results, we follow an approach outlined on the Gaia DR2 website $^{6}$ to determine the variance $\sigma_{\mathrm{h}}^{2}$ of the mean proper motion of a star cluster. According to this approach, the variance has a random and a systematic component and can be calculated according to the following formula for a sample of $n$ stars that have proper motion uncertainties $\sigma_{\mu, i}$.

$\sigma_{\mathrm{h}}^{2}=\frac{1}{n}\left(\frac{k}{n} \sum_{i=1}^{n} \sigma_{\mu, i}^{2}+V_{\mu}(0)\right)+\frac{2}{n^{2}} \sum_{i=2}^{n} \sum_{j=1}^{i-1} V_{\mu}\left(\phi_{i, j}\right)$.

$k$ is a correction factor for the uncertainties provided in DR2 that we set to 1.0. The spatial covariance function $V_{\mu}$ has been derived by Lindegren et al. (2018) and can be retrieved from the Gaia webpage. It depends on the angular distance $\phi_{i, j}$ between two sources, given in degrees. The second, systematic term in equation (4) is expected to dominate the error budget for large sample sizes.

Using the positions and proper motion uncertainties of our sample stars in both clusters, we found that the systematic errors affecting the proper motion means of the clus-

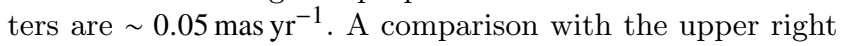
panel of Fig. 5 shows that this potential systematic error is larger than the apparent contraction visible in the dataset of NGC 6819. This indicates that with the current Gaia data it is not possible to verify if the observed trend is intrinsic or spurious.

6 https://www. cosmos.esa.int/web/gaia/dr2-known-issues, see link to presentation by Lindegren et al. 
The potential systematic errors are also comparable to the rotation signal that we detect in NGC 6791 (cf. upper left panel in Fig. 5). However, in this case it is reassuring that a rotation signal of similar strength is observed in the LOS velocity sample. Therefore, we do not expect that the rotation signal in NGC 6791 is caused by systematics in the data, although a final judgment will only be possible with future Gaia data releases.

\section{CONCLUSIONS}

In this work, we combined Gaia data with archival LOS velocities to study the internal dynamics of two old open clusters, NGC 6791 and NGC 6819, in three dimensions. For both clusters, an alignment of stellar spins has recently been reported (Corsaro et al. 2017), suggesting that they inherited significant angular momentum from their parent gas clouds during formation. Such a transfer of angular momentum should also leave a fingerprint in the internal kinematics of the clusters and the aim of our study was to search for such fingerprints.

We found the clusters to have remarkably different kinematics. While both the LOS velocity and the proper motion data reveal clear signs of rotation in NGC 6791, no such signatures are found in NGC 6819 . The rotation velocities we measure in NGC 6791 are $\sim 0.5 \mathrm{~km} \mathrm{~s}^{-1}$ whereas our upper limits in NGC 6819 are $\lesssim 0.1 \mathrm{~km} \mathrm{~s}^{-1}$. Both clusters have velocity dispersions $\sim 1 \mathrm{~km} \mathrm{~s}^{-1}$, hence rotation seems far more important in NGC 6791 than in NGC 6819.

Star clusters experience significant mass loss due to tidal forces or their dynamical evolution. Stars escaping the clusters carry angular momentum, so that primordial rotation fields are expected to decrease with time (e.g. Einsel \& Spurzem 1999). We used eq. (7.108) in Binney \& Tremaine (2008) to estimate relaxation times of $560 \mathrm{Myr}$ (NGC 6791) and $260 \mathrm{Myr}$ (NGC 6819), respectively. Hence, both clusters have cycled through about 10 relaxation times during their lives. Therefore, it is perhaps more surprising that NGC 6791 rotates than that NGC 6819 does not. One possibility is that NGC 6819 formed more compact and has therefore undergone more relaxation than the estimate from above.

We note that NGC 6791 is more massive than NGC $6819\left(5000 \mathrm{M}_{\odot}\right.$ compared to $\left.2600 \mathrm{M}_{\odot}\right)$. Furthermore, Dalessandro et al. (2015) suggest that NGC 6791 was born with a much higher mass of $(1.5-4) \times 10^{5} \mathrm{M}_{\odot}$, although note that the initial mass of the $N$-body model of NGC 6791 by Martinez-Medina et al. (2018) was much lower $\left(5 \times 10^{4} \mathrm{M}_{\odot}\right)$. A possibility to explain the differences in the dynamics of the two clusters may therefore be that rotation plays a more important role in high-mass clusters. Such a mass dependency could explain why rotation is observed in massive clusters of all ages (e.g. Hénault-Brunet et al. 2012; Davies et al. 2011; Kamann et al. 2018a) while Kuhn et al. (2018) did not observe rotation in the young low-mass clusters of their sample.

The observed rotation velocities in NGC 6791 suggest a specific angular momentum comparable to that of rotating globular clusters with similar ages but much longer relaxation times (Kamann et al. 2018a; Bianchini et al. 2018). A possible explanation - especially considering the mass loss that NGC 6791 likely experienced - could be that part of the rotation signal is not primordial but has been introduced by tidal forces (e.g. Claydon et al. 2017; Lanzoni et al. 2018). Tiongco et al. (2016) found that in such cases a cluster would be characterized by solid-body rotation (i.e. constant angular velocities), which seems in line with the behaviour we observe in NGC 6791 (cf. Fig. 5).

Given the properties of NGC 6791 (mass, size, orbit), tidally induced rotation is expected to become important at radii $\gtrsim 10^{\prime}$. In both datasets, LOS velocities and proper motions, we observe that rotation sets in further inwards. Nevertheless, we note that in the proper motion sample, we only find a significant rotation signal beyond $\sim 4^{\prime}$. But tides give rise to a net retrograde rotation (in a frame corotating with the cluster orbit) with respect to the orbit (see Claydon et al. 2017, and references therein). Because the line-of-sight to NGC 6791 is approximately parallel to its orbital plane, this implies that they impact the LOS velocities stronger than the proper motions.

We determine an inclination angle of $i=45_{-18}^{+11}$ degrees for the cluster rotation field in NGC 6791. Taken at face value, it suggests that the cluster rotates in a plane that is titled with respect to the mean stellar spin orientation found by Corsaro et al. (2017). However, the uncertainties are still considerable, both for the stellar spin measurements (e.g. Kamiaka et al. 2018) and for our constraint on $i$. In addition, if the rotation field of the cluster is shaped by tidal forces, its orientation will likely be different from any primordial rotation field. Nevertheless, NGC 6791 occurs as a promising target for follow-up studies on a possible link between cluster rotation and stellar spins using future Gaia data releases and potentially larger LOS velocity samples. In addition, we plan to extend our studies to younger clusters where the effects of relaxation only play a minor role. In light of the findings by Kovacs (2018), M44 might be a promising targets as it is significantly younger, but also less massive, than NGC 6791 and NGC 6819.

\section{ACKNOWLEDGEMENTS}

We thank the anonymous referee for a timely and well justified report that helped us to improve the paper. We thank Joel Pfeffer for enlightening discussions during the preparation of the manuscript. SK and NB gratefully acknowledge funding from a European Research Council consolidator grant (ERC-CoG-646928-Multi-Pop). MG acknowledges financial support from the Royal Society (University Research Fellowship) and MG and EB thank the European Research Council (ERC StG-335936, CLUSTERS) for financial support. EB acknowledges partial support by a Vici grant from the Netherlands Organisation for Scientific Research (NWO). VHB acknowledges support from the NRCCanada Plaskett Fellowship. We also thank the International Space Science Institute (ISSI, Bern, CH) for welcoming the activities of the Team 407 "Globular Clusters in the Gaia Era" (team leaders VHB \& MG), during which part of this work was conducted. This research has made use of the SIMBAD database, operated at CDS, Strasbourg, France (Wenger et al. 2000). This work has made use of data from the European Space Agency (ESA) mission Gaia (https: //www.cosmos.esa.int/gaia), processed by the Gaia Data Processing and Analysis Consortium (DPAC, https://www. 
cosmos.esa.int/web/gaia/dpac/consortium). Funding for the DPAC has been provided by national institutions, in particular the institutions participating in the Gaia Multilateral Agreement.

\section{REFERENCES}

Basu S., et al., 2011, ApJ, 729, L10

Bedin L. R., Piotto G., Carraro G., King I. R., Anderson J., 2006, A\&A, 460, L27

Bellazzini M., Bragaglia A., Carretta E., Gratton R. G., Lucatello S., Catanzaro G., Leone F., 2012, A\&A, 538, A18

Bianchini P., van der Marel R. P., del Pino A., Watkins L. L., Bellini A., Fardal M. A., Libralato M., Sills A., 2018, MNRAS, 481, 2125

Binney J., Tremaine S., 2008, Galactic Dynamics: Second Edition. Princeton University Press

Bragaglia A., et al., 2001, AJ, 121, 327

Cappellari M., 2002, MNRAS, 333, 400

Carraro G., Villanova S., Demarque P., McSwain M. V., Piotto G., Bedin L. R., 2006, ApJ, 643, 1151

Chen W. P., Chen C. W., Shu C. G., 2004, AJ, 128, 2306

Choi J., Conroy C., Ting Y.-S., Cargile P. A., Dotter A., Johnson B. D., 2018, ApJ, 863, 65

Claydon I., Gieles M., Zocchi A., 2017, MNRAS, 466, 3937

Corsaro E., et al., 2017, Nature Astronomy, 1, 0064

Dalessandro E., Miocchi P., Carraro G., Jílková L., Moitinho A., 2015, MNRAS, 449, 1811

Dalgleish H. S., Longmore S. N., Peters T., Henshaw J. D., VeitchMichaelis J. L., Urquhart J. S., 2018, MNRAS, 478, 3530

Daniel K. J., Heggie D. C., Varri A. L., 2017, MNRAS, 468, 1453

Davies B., Bastian N., Gieles M., Seth A. C., Mengel S., Konstantopoulos I. S., 2011, MNRAS, 411, 1386

Einsel C., Spurzem R., 1999, MNRAS, 302, 81

Evans N. W., de Zeeuw P. T., 1994, MNRAS, 271, 202

Fabricius M. H., et al., 2014, ApJ, 787, L26

Ferraro F. R., et al., 2018, ApJ, 860, 50

Foreman-Mackey D., 2016, The Journal of Open Source Software, 24

Foreman-Mackey D., Hogg D. W., Lang D., Goodman J., 2013, PASP, 125, 306

Gaia Collaboration et al., 2016, A\&A, 595, A1

Gaia Collaboration et al., 2018a, preprint, (arXiv:1804.09381)

Gaia Collaboration et al., 2018b, A\&A, 616, A1

Grundahl F., Clausen J. V., Hardis S., Frandsen S., 2008, A\&A, 492, 171

Hénault-Brunet V., et al., 2012, A\&A, 545, L1

Hénault-Brunet V., Gieles M., Agertz O., Read J. I., 2015, MNRAS, 450, 1164

Hole K. T., Geller A. M., Mathieu R. D., Platais I., Meibom S., Latham D. W., 2009, AJ, 138, 159

Jílková L., Carraro G., Jungwiert B., Minchev I., 2012, A\&A, 541, A64

Kalirai J. S., et al., 2001, AJ, 122, 266

Kamann S., et al., 2018a, MNRAS, 473, 5591

Kamann S., et al., 2018b, MNRAS, 480, 1689

Kamiaka S., Benomar O., Suto Y., 2018, MNRAS, 479, 391

Klagyivik P., et al., 2013, ApJ, 773, 54

Kovacs G., 2018, A\&A, 612, L2

Kuhn M. A., Hillenbrand L. A., Sills A., Feigelson E. D., Getman K. V., 2018, preprint, (arXiv:1807.02115)

Küpper A. H. W., Kroupa P., Baumgardt H., Heggie D. C., 2010, MNRAS, 407, 2241

Lanzoni B., et al., 2018, ApJ, 865, 11

Lee-Brown D. B., Anthony-Twarog B. J., Deliyannis C. P., Rich E., Twarog B. A., 2015, AJ, 149, 121
Lindegren L., et al., 2018, A\&A, 616, A2

Martinez-Medina L. A., Gieles M., Pichardo B., Peimbert A., 2018, MNRAS, 474, 32

Mathieu R. D., 2000, in Pallavicini R., Micela G., Sciortino S., eds, Astronomical Society of the Pacific Conference Series Vol. 198, Stellar Clusters and Associations: Convection, Rotation, and Dynamos. p. 517

Milone A. P., Marino A. F., Mastrobuono-Battisti A., Lagioia E. P., 2018, MNRAS, 479, 5005

Peebles P. J. E., 1969, ApJ, 155, 393

Platais I., Cudworth K. M., Kozhurina-Platais V., McLaughlin D. E., Meibom S., Veillet C., 2011, ApJ, 733, L1

Platais I., Gosnell N. M., Meibom S., Kozhurina-Platais V., Bellini A., Veillet C., Burkhead M. S., 2013, AJ, 146, 43

Portegies Zwart S. F., McMillan S. L. W., Hut P., Makino J., 2001, MNRAS, 321, 199

Rey-Raposo R., Read J. I., 2018, MNRAS, 481, L16

Rosvick J. M., Vandenberg D. A., 1998, AJ, 115, 1516

Terlevich E., 1987, MNRAS, 224, 193

Tiongco M. A., Vesperini E., Varri A. L., 2016, MNRAS, 461, 402 Tofflemire B. M., Gosnell N. M., Mathieu R. D., Platais I., 2014, AJ, 148,61

Wenger M., et al., 2000, A\&AS, 143, 9

Zahn J.-P., 1977, A\&A, 57, 383

Zocchi A., Gieles M., Hénault-Brunet V., Varri A. L., 2016, MNRAS, 462, 696

van Leeuwen F., Le Poole R. S., Reijns R. A., Freeman K. C., de Zeeuw P. T., 2000, A\&A, 360, 472

van de Ven G., van den Bosch R. C. E., Verolme E. K., de Zeeuw P. T., 2006, A\&A, 445, 513

van den Bergh S., 2008, AJ, 135, 1731

\section{APPENDIX A: ANALYSIS OF MOCK DATA}

To verify if our approach can be used to get a useful constraint on the inclination angle in NGC 6791, we used a simulated cluster from the study of Hénault-Brunet et al. (2015). The data is based on the final snapshot of an $N$ body model of a cluster evolved to an age of $10.75 \mathrm{Gyr}$, for which the initial dimensionless spin parameter $\lambda$ (Peebles 1969) was set to $\lambda=0.091$ (model MGEN1 in Table 1 of Hénault-Brunet et al. 2015), corresponding to about $10 \%$ of the total kinetic energy of the cluster in rotation at the start of the simulation.

We scaled the simulation to the properties of NGC 6791 and extracted LOS velocity and proper motion samples from the simulation. This was done under the assumption that the rotation axis of the cluster is observed at a rotation axis angle of $\theta_{0}=-153 \mathrm{deg}$. (measured north through east) and at an inclination of $i=53 \mathrm{deg}$.. The samples were chosen such that they had the same numbers of stars and the same radial distributions as the observed data of NGC 6791. Each velocity drawn from the simulation was then assigned the uncertainty of an actually observed star and scattered accordingly. Then, we performed the same analysis on the mock data as we did for the actual data of NGC 6791 .

The results obtained for the LOS velocity data are summarized in Fig. A1. It is obvious that the posterior probability distributions obtained by MCMC sampling show clear evidence for a rotating cluster. We obtain a rotation axis angle of $\theta_{0}=-146 \pm 19 \mathrm{deg}$., which is consistent with the rotation axis angle used to create the mock sample. We further obtain an amplitude of $v_{\text {rot }}=0.51 \pm 0.17 \mathrm{~km} \mathrm{~s}^{-1}$ for the rotation velocity. 


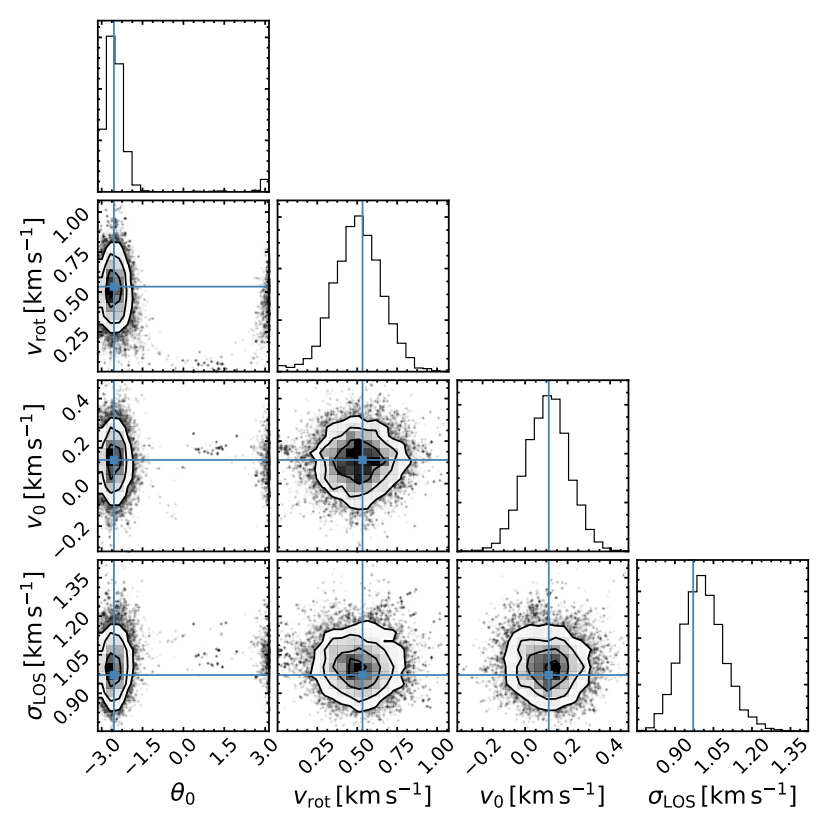

Figure A1. The same as Fig. 4 for the mock data resembling NGC 6791.

In the next step, we repeated the analysis performed for the proper motions. Fig. A2 shows radial profiles of the mean velocity and the velocity dispersion for the tangential and the radial components of the proper motion. The tangential component shows clear signs of counterclockwise rotation, indicated by positive mean values. For the whole sample, we obtain a mean of $\mu_{\mathrm{t}}=0.66 \pm 0.10 \mathrm{~km} \mathrm{~s}^{-1}$ using the assumed distance of $4.92 \mathrm{kpc}$. A direct comparison to the rotation amplitude obtained from the line of sight velocity data yields an estimate of the inclination angle of $i=38 \pm 10 \mathrm{deg}$. which is $2 \sigma$ off from the true value of 53 degrees.

Next, we checked whether we can use eq. 3 to obtain a more physically motivated estimate of the inclination angle. To this aim, we transformed our data to a coordinate system $(u, v)$, where the $v$-axis is aligned with the semi-minor axis (or rotation axis) of the system found in the analysis of the LOS velocities. Afterwards, we binned the data on a polar grid (after exploiting the symmetry relations from Sect. 4.3 to transform all measurements to the first quadrant) such that each grid point contained at least 10 LOS velocity measurements. Then, we obtained mean values for the LOS velocity and both proper motion components in each bin. The relation between the mean LOS velocity $\left(\left\langle v_{\mathrm{r}}\right\rangle\right)$ and the mean of the minor-axis proper motion $\left(\left\langle\mu_{\mathrm{v}}\right\rangle\right)$ for the individual bins is shown in Fig. A3. Again, the blue line shows a linear fit to the data, performed with an orthogonal distance regression method that accounts for the uncertainties along both axes. Using equation (3), we obtain an inclination angle of $i=51_{-10}^{+7}$ degress, in good agreement with our input value of $i=54$ degrees. Hence we are confident that the inclination angle determined this way for the actual data of NGC 6791 is robust.

This paper has been typeset from a $\mathrm{T}_{\mathrm{E}} \mathrm{X} / \mathrm{LAT}_{\mathrm{E}} \mathrm{X}$ file prepared by the author.
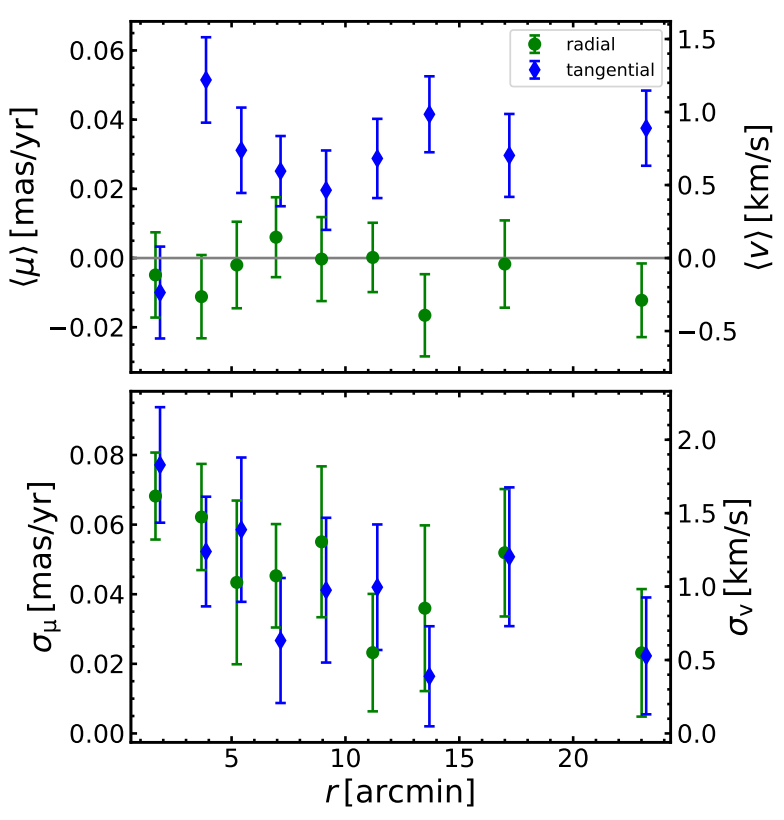

Figure A2. The same as Fig. 5 for the mock data resembling NGC 6791.

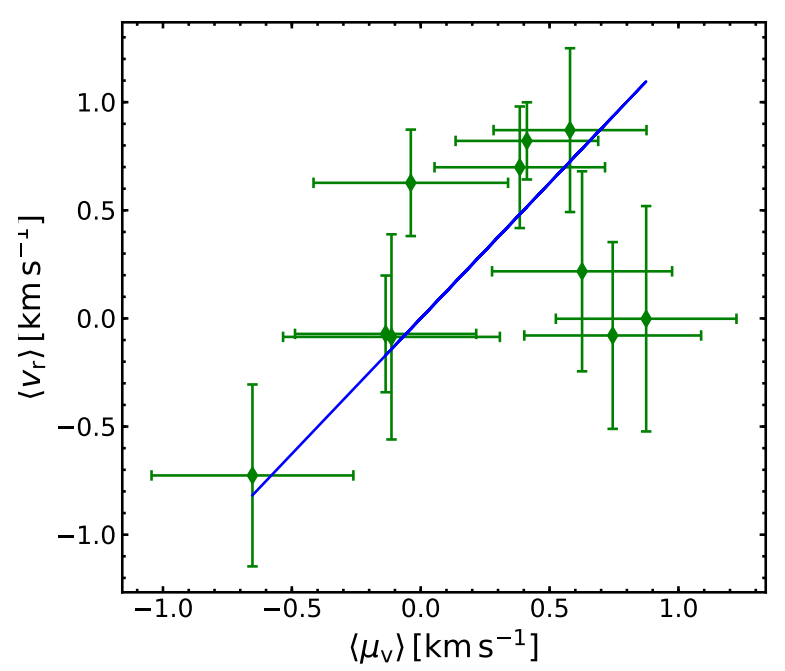

Figure A3. As in Fig. 6, we show the relation between the mean of the LOS velocity and the mean of the proper motion component along the semi-minor axis in different spatial bins of the mock system. The blue line shows a linear fit to the data using an orthogonal distance regression method that takes into account the uncertainties in both coordinates. It was forced to go through the origin. 